\title{
MONTE CARLO SIMULATION OF Ly $\alpha$ SCATTERING AND APPLICATION TO DAMPED Ly $\alpha$ SYSTEMS
}

\author{
Zheng Zheng AND Jordi Miralda-Escudé \\ Department of Astronomy, Ohio State University, 140 West 18th Avenue, Columbus, OH 43210; \\ zhengz@astronomy.ohio-state.edu,jordi@astronomy.ohio-state.edu \\ Received 2002 March 15; accepted 2002 June 18
}

\begin{abstract}
A Monte Carlo code to solve the transfer of Ly $\alpha$ photons is developed that can predict the Ly $\alpha$ image and two-dimensional Ly $\alpha$ spectra of a hydrogen cloud with any given geometry, Ly $\alpha$ emissivity, neutral hydrogen density distribution, and bulk velocity field. We apply the code to several simple cases of a uniform cloud to show how the $\operatorname{Ly} \alpha$ image and emitted line spectrum are affected by the column density, internal velocity gradients, and emissivity distribution. We then apply the code to two models for damped Ly $\alpha$ absorption systems: a spherical, static, isothermal cloud and a flattened, axially symmetric, rotating cloud. If the emission is due to fluorescence of the external background radiation, the Ly $\alpha$ image should have a core corresponding to the region where hydrogen is self-shielded. The emission-line profile has the characteristic double peak with a deep central trough. We show how rotation of the cloud causes the two peaks to shift in wavelength as the slit is perpendicular to the rotation axis and how the relative amplitude of the two peaks is changed. In reality, damped Ly $\alpha$ systems are likely to have a clumpy gas distribution with turbulent velocity fields, which should smooth the line emission profile but should still leave the rotation signature of the wavelength shift across the system.
\end{abstract}

Subject headings: line: formation — quasars: absorption lines — radiative transfer — scattering

\section{INTRODUCTION}

The high column density absorption systems (damped Ly $\alpha$ and Lyman limit systems) are a key part of understanding galaxy formation. As a galaxy collapses from the highly ionized intergalactic medium, the gas will inevitably go through a phase of a self-shielded cloud of atomic hydrogen before it can cool and collapse further into molecular clouds, form stars, and increase its metallicity. Many of the damped Ly $\alpha$ systems (DLAs) at high redshift may be gaseous halos in the process of forming galaxies rather than fully formed spiral disks, especially in view of the very low metallicities at high redshifts (Lu et al. 1996; Prochaska, Gawiser, \& Wolfe 2001). If so, the internal structure of these objects should reveal to us the detailed processes by which galaxies form. Several basic questions arise in relation to this: How big are the absorption systems? Is the gas smoothly distributed or is it in the form of clumps and an interclump medium? If so, how big are the clumps? What is the dynamical state of the gas? How does its mean rotation velocity compare to its velocity dispersion?

Some of these questions may be addressed by studying the associated metal absorption lines (see, e.g., Prochaska \& Wolfe 1997, 1998) or by radio and optical observations of galaxies found to be associated with the absorbers (see Briggs et al. 1989; Djorgovski et al. 1996), although the majority of DLAs at high redshifts might not be associated with luminous galaxies.

An alternative observational probe of the structure of DLAs and Lyman limit systems (LLSs) may be found in their Ly $\alpha$ emission produced in hydrogen recombinations and the subsequent scattering of Ly $\alpha$ photons through the gas (Hogan \& Weymann 1987; Gould \& Weinberg 1996). The source of the ionization may be the external cosmic ionizing radiation, shock heating due to the gravitational collapse of the gas in a dark matter halo, or star formation within the system. In the case of fluorescence of external radiation, the maximum surface brightness is achieved in any system with $N_{\mathrm{H}_{\mathrm{I}}} \gtrsim 10^{18} \mathrm{~cm}^{-2}$, where all the incident external photons are absorbed.

Efforts have been made to image known DLAs and LLSs toward high-z quasars. DLAs toward PKS 0528-250 (Warren \& Møller 1996) and Q0151+048A (Fynbo, Møller, \& Warren 1999) and one LLS toward Q1205-30 (Fynbo, Thomsen, \& Warren 2000) have been successfully detected using tuned narrowband filters. There are also some spectroscopically confirmed detections of Ly $\alpha$ emission from DLAs (see, e.g., Hunstead, Pettini, \& Fletcher 1990; Djorgovski 1998; Pettini et al. 1995; Leibundgut \& Robertson 1999). Most of these absorber systems with Ly $\alpha$ emission detected are near the redshift of the background quasar, which suggests a $\operatorname{Ly} \alpha$ source due to photoionization by the quasar (Fynbo et al. 1999, 2000).

The hydrogen Ly $\alpha$ line is a resonance line. The problem of radiative transfer of resonance-line radiation can be approximately solved analytically under certain conditions (see, e.g., Harrington 1973, 1974; Neufeld 1990). These analytic solutions can be found only for a limited number of cases such as a static, extremely opaque and plane-parallel medium. In some cases, numerical methods are used to solve the transfer equation (see, e.g., Adams 1972; Hummer \& Kunasz 1980; Urbaniak \& Wolfe 1981). For a more general geometry, density distribution, and kinematics, the Monte Carlo method turns out to be very useful, and thus, it has been applied to many problems (see, e.g., Auer 1968; Avery \& House 1968; Caroff, Noerdlinger, \& Scargle 1972; Panagia \& Ranieri 1973; Bonilha et al. 1979; Natta \& Beckwith 1986; Ahn, Lee, \& Lee 2000, 2001, 2002).

This paper presents the results of a numerical Monte Carlo code we have developed to compute the transfer of Ly $\alpha$ photons through an arbitrary distribution of hydrogen. The code predicts the two-dimensional image and line spectrum along any given observed direction, for a general three-dimensional distribution of gas of given geometry, 
Ly $\alpha$ emissivity, neutral hydrogen density, and bulk velocity field. In this paper, it will be applied only to spherical and axially symmetric clouds, although in the future it can be applied to results of numerical simulations of galaxy formation.

We describe the Monte Carlo code in $\S 2$. In $\S 3$, we apply the code to several simple cases of hydrogen clouds to demonstrate the effects of the neutral hydrogen column density, Ly $\alpha$ emissivity, and bulk velocity field on the spectra of escaped Ly $\alpha$ photons. In $\S 4$, we model DLAs as static and rotating clouds and investigate their two-dimensional Ly $\alpha$ emission spectra. Finally, we have a brief summary and discussion in $\S 5$.

\section{MONTE CARLO SIMULATION OF Ly $\alpha$ SCATTERING}

\subsection{Description of the Method}

The method used to numerically compute the images and spectra of $\operatorname{Ly} \alpha$ emission consists of generating random realizations of the trajectory of a large number of Ly $\alpha$ photons as they are scattered within the specified gas distribution. In a nutshell, the method proceeds through the following steps: First, we generate the point where the photon is created, with the emissivity distribution proportional to the recombination rate at every point. Second, the optical depth $\tau$ that the photon will travel through before it is scattered is randomly generated with a probability density $e^{-\tau}$. The spatial location of the scattering at optical depth $\tau$ from the point of emission is then determined along a randomly chosen direction, with the knowledge of the neutral hydrogen density distribution and the scattering cross section (see eq. [2]). We then generate the velocity of the atom that scatters the photon, as described below, compute the new frequency of the photon after scattering, and generate the new direction of the photon. The process of propagation and scattering is repeated until the photon escapes the modeled system.

As these random realizations of photon trajectories are being carried out, a Ly $\alpha$ image of the system along a specified direction can be created. The image consists of a threedimensional array of the two projected coordinates perpendicular to the direction of the image and the frequency of the escaped photons. The array contains the mean number of photons emitted at every projected position and frequency. At every scattering of a photon, the probability that the photon is reemitted in the direction of the image and escapes is separately calculated. This probability is added on the element of the image array corresponding to the projected position and frequency of the photon. In practice, for the majority of the scatterings, the optical depth is large and the probability of escape is negligible, so one can avoid the computation of the optical depth in the direction of the image on most scatterings.

We now describe how the optical depth of the Ly $\alpha$ photons is computed and how the velocity of the atom at each scattering is generated. The scattering cross section of Ly $\alpha$ photons as a function of the frequency in the rest frame of the hydrogen atom is

$$
\sigma_{\nu}=f_{12} \frac{\pi e^{2}}{m_{e} c} \frac{\Delta \nu_{\mathrm{L}} / 2 \pi}{\left(\nu-\nu_{0}\right)^{2}+\left(\Delta \nu_{\mathrm{L}} / 2\right)^{2}},
$$

where $f_{12}=0.4162$ is the Ly $\alpha$ oscillator strength, $\nu_{0}=2.466 \times 10^{15} \mathrm{~Hz}$ is the line-center frequency, $\Delta \nu_{\mathrm{L}}=4.03 \times 10^{-8} \nu_{0}=9.936 \times 10^{7} \mathrm{~Hz}$ is the natural line width, and the other symbols have their usual meaning. For a hydrogen atom with a velocity component $v_{z}$ along the photon's direction in a fixed "laboratory" frame, the term $\nu-\nu_{0}$ in the above equation becomes $\left(\nu_{i}-\nu_{0}\right)-\left(\nu_{z} / c\right) \nu_{0}$, where $\nu_{i}$ is the frequency of the incident photon in the laboratory frame. For a Maxwellian distribution of atom velocities, the resulting average cross section is

$$
\sigma\left(x_{i}\right)=f_{12} \frac{\sqrt{\pi} e^{2}}{m_{e} c \Delta \nu_{\mathrm{D}}} H\left(a, x_{i}\right),
$$

where

$$
H(a, x)=\frac{a}{\pi} \int_{-\infty}^{+\infty} \frac{e^{-y^{2}}}{(x-y)^{2}+a^{2}} d y
$$

is the Voigt function, $\Delta \nu_{\mathrm{D}}=\left(v_{p} / c\right) \nu_{0}$ is the Doppler frequency width, $v_{p}=\left(2 k T / m_{\mathrm{H}}\right)^{1 / 2}$ is the atom velocity dispersion times $\sqrt{2}, T$ is the gas temperature, $m_{\mathrm{H}}$ is the mass of the hydrogen atom, $x_{i}=\left(\nu_{i}-\nu_{0}\right) / \Delta \nu_{\mathrm{D}}$ is the relative frequency of the incident photon in the laboratory frame, and $a=\Delta \nu_{\mathrm{L}} /\left(2 \Delta \nu_{\mathrm{D}}\right)$ is the relative line width. The optical depth along the photon trajectory is computed by integrating the cross section in equation (2) times the atomic hydrogen density.

Once a spatial location where the photon is scattered has been determined, the velocity $v_{z}$ along the direction of the incident photon of the atom responsible for the scattering obeys the following distribution:

$$
f\left(u_{z}\right)=\frac{a}{\pi} \frac{e^{-u_{z}^{2}}}{\left(x_{i}-u_{z}\right)^{2}+a^{2}} H^{-1}\left(a, x_{i}\right),
$$

where $u_{z}=v_{z} / v_{p}$. We describe how we generate random numbers with this distribution in the Appendix. The two velocity components perpendicular to the direction of the photon simply follow a Gaussian distribution parameterized by the local temperature.

After the total velocity of the atom is chosen according to the above distribution, we first perform a Lorentz transform of the direction and frequency of the photon to the rest frame of the atom. The direction of the scattered photon is then generated according to a dipole distribution in this frame. Its frequency differs from the incident one by the recoil effect, which is taken into account in the code (it is negligibly small in the applications we will present in this paper). The direction and frequency of the scattered photon are then transformed back to the laboratory frame, a new optical depth is chosen, and the entire process is repeated until the photon escapes from the cloud. In the presence of a fluid velocity, the procedure changes only by replacing the incident frequency $x_{i}$ in equations (2) and (4) by its value in the fluid frame, $x_{f i}=x_{i}-\left(v_{f z} / c\right)\left(\nu_{0} / \Delta \nu_{\mathrm{D}}\right)$, where $v_{f z}$ is the fluid velocity parallel to the photon's direction (valid in the nonrelativistic regime).

As mentioned previously, the Ly $\alpha$ image and spectrum of the system are calculated as the photon trajectories are randomly generated. At every photon scattering, we compute first the optical depth for the photon to escape the cloud along the direction of the image, and the photon is added to the corresponding element of the image array in projected 
position and frequency, with the weight $e^{-\tau}\left(1+\mu^{2}\right) d \Omega$, where $\tau$ is the optical depth for escaping, $\mu$ is the cosine of the angle between the incident photon and the image direction, and $d \Omega$ is the solid angle subtended by the pixel (both $\mu$ and $d \Omega$ are measured in the rest frame of the atom). The factor $1+\mu^{2}$ accounts for the dipole probability distribution of the direction of the photon after scattering. A photon also has a probability to directly escape the system along the direction of the image when it is created, which is added to the corresponding element of the image array. After the contribution to the image has been added, a random direction of the new scattered photon is generated according to the same dipole distribution, and the realization of the photon trajectory is continued until the photon finally escapes from the cloud. The total number of photons in the simulation is chosen to give an acceptable signal-to-noise ratio (the typical number of photons used for simulations in this paper is on the order of $\sim 10^{3}-10^{4}$ ).

\subsection{Tests of the Code}

We test our numerical code for the case of a static, planeparallel slab, for which Neufeld (1990) derived an analytic solution of the mean intensity in the limit of a large optical depth. We simulate the case of a midplane source radiating line-center Ly $\alpha$ photons in a plane-parallel slab of uniform density for three optical depths: $\tau_{0}=10^{4}, 10^{5}$, and $10^{6}$, where $\tau_{0}=\sigma\left(x_{i}=0\right) N_{\mathrm{H}}$ is the line-center optical depth from the midplane to the boundary of the slab. We assume a temperature $T=10 \mathrm{~K}\left(a \approx 1.49 \times 10^{-2}\right)$. As we have mentioned, the recoil effect is taken into account in the code. For the purpose of comparison, we first turn off this effect and run the simulation. We compare the spectra of the escaped Ly $\alpha$ photons with the analytic results (eq. [2.24] in Neufeld 1990; note that his definition of $\tau_{0}$ differs from ours by a factor of $\sqrt{\pi}$ as $a \ll 1$; see also Ahn et al. 2001). The left-hand panel of Figure 1 shows excellent agreement, becoming better as the optical depth increases as expected since the analytic solution applies in the limit of a large optical depth $\left(\sqrt{\pi} \tau_{0} \gtrsim 10^{3} / a\right.$; Neufeld 1990). We then turn on the recoil effect and rerun the simulation. The results are shown in the right-hand panel of Figure 1. The double-peaked frequency distribution of the escaped photons becomes asymmetricmore photons appear at the red part. The profile from analytic solutions can be regarded as an average of the red part and the blue part. The magnitude of the recoil effect is easily understood as reflecting the thermalization of photons around frequency $\nu_{0}$ inside the cloud (Wouthuysen 1952; Field 1959), which modifies their abundance by a factor of $e^{-x / x_{T}}$, where $x \equiv \Delta \nu / \Delta \nu_{\mathrm{D}}$ and $x_{T} \equiv k T /\left(h \Delta \nu_{\mathrm{D}}\right)$ $=\left(m_{\mathrm{H}} c^{2} k T / 2\right)^{1 / 2} /\left(h \nu_{0}\right)$. Obviously, the recoil effect becomes more important for low temperature and large optical depth.

We also test the code for a spherically symmetric case with a particular bulk velocity field. Loeb \& Rybicki (1999) study and simulate the scattered $\mathrm{Ly} \alpha$ radiation around a point source before cosmological reionization. The Ly $\alpha$ photons are scattered by the intergalactic medium, which is undergoing Hubble expansion. According to their approximation, the gas temperature is regarded as zero. We run a corresponding simulation with our code and find that the frequency distribution and the surface brightness profile of the escaped Ly $\alpha$ photons shown in Loeb \& Rybicki (1999) are well reproduced.

\section{SPHERICAL CLOUDS OF UNIFORM DENSITY}

We start applying our code to the most simple case of spherical hydrogen clouds with uniform density. We consider the following cases:

case 1.- uniform emissivity, static cloud;

case 2.- uniform emissivity, expanding or contracting cloud;

case 3. - central point source, static cloud;

case 4.-central point source, expanding or contracting cloud.

For each case, we perform two runs with different column densities $\left(2 \times 10^{18}\right.$ and $2 \times 10^{20} \mathrm{~cm}^{-2}$, corresponding to line-center optical depths $\tau_{0}=8.3 \times 10^{4}$ and $8.3 \times 10^{6}$, respectively). The temperature is assumed to be $2 \times 10^{4} \mathrm{~K}$ everywhere. In the case of expansion, we set it to be Hubblelike (i.e., the velocity is proportional to the radius), with the velocity at the edge of the system fixed to be $200 \mathrm{~km} \mathrm{~s}^{-1}$. The cases of contraction are done in the same way; their line spectra can generally be obtained by simply mirrorreflecting in frequency the photons that escaped from the expanding cloud about the rest-frame Ly $\alpha$ frequency.

Figures 2 and 3 show the distribution of escaped photons for all the cases. For static cases, as expected, the escaped photons have a double-peaked frequency distribution. Except for the very small effect of atomic recoil (see Field 1959), the two peaks are exactly symmetric; the differences in the figures are due to the simulation noise.

The results obtained for the emergent spectrum can be understood by noting the typical trajectory in frequency and space followed by a photon (see, e.g., Osterbrock 1962; Adams 1972; Urbaniak \& Wolfe 1981; Ahn et al. 2000). In the case of an LLS (like in Fig. 2), the photons are always most likely to be scattered by atoms that have the right velocity along the photon direction so that, in their rest frame, the photon frequency is shifted very close to the line center. This implies that there is practically no correlation between the frequency of the photon before and after scattering. At any random time, a photon is most likely to be found within a frequency range $\Delta \nu_{\mathrm{D}}=\left(v_{p} / c\right) \nu_{0}$ of the line center and will occasionally make larger excursions away from the line center when scattered by an atom having a large velocity perpendicular to the photon's direction. After any such excursion, the photon will likely return immediately to the line center with one or a few scatterings. Therefore, the photon escapes the cloud not by diffusing in frequency but by making a single large jump when scattered by an atom in the high-velocity tail of the Maxwellian distribution, which reduces its optical depth for escaping to a value of order unity. For an LLS with $N_{\mathrm{H}_{\mathrm{I}}}=2 \times 10^{18} \mathrm{~cm}^{-2}$, the line-center optical depth is $\tau_{0}=8.3 \times 10^{4}$, and the photons escape when their optical depth is $\tau=\tau_{0} e^{-x^{2}} \sim 1$ (where $\left.x \equiv \Delta \nu / \Delta \nu_{\mathrm{D}}\right)$, which implies $|x| \simeq\left(\ln \tau_{0}\right)^{1 / 2}=3.4$.

In the case of a DLA, the scattering history differs from the Lyman limit case once the photon reaches a sufficiently large excursion in frequency to make it more likely that the next scattering is caused by a random atom far from the line center rather than an atom moving with the right velocity to shift the photon frequency to the line center in the atom frame. Then the evolution of the photon is described by diffusion in frequency. Since the photons are now reaching their escaping frequency not by a large jump but by a series of small steps, 

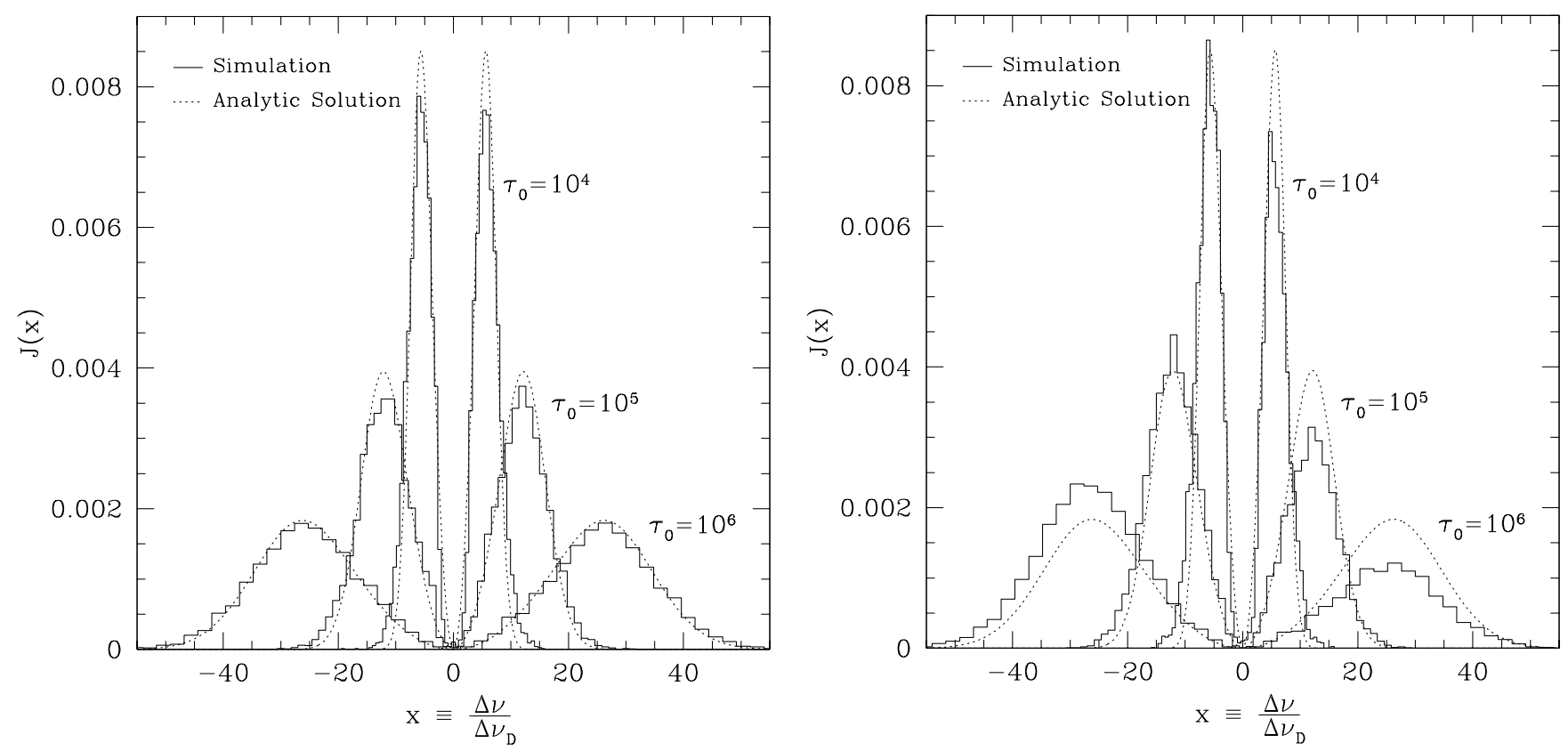

FIG. 1.-Comparison between results from our Monte Carlo simulations and the analytic solutions. The dotted lines are the analytic solutions (Neufeld 1990) of the Ly $\alpha$ spectra at one boundary of a slab with a midplane source and different scattering optical depths. The solid lines are those from simulations. In the left-hand panel, the recoil effect is neglected in the simulations; in the right-hand panel, this effect is taken into account in the simulations (see text).

they undergo greater spatial diffusion than in LLSs. This, plus the fact that the optical depth now has a power-law instead of Gaussian dependence at large $x$, broadens the width of the two emission peaks. Of course, the emission peaks also move farther from the line center because a smaller scattering cross section is required for the photons to escape.

In Figure 4, we can see that the surface brightness produced by a central point source is more extended for a DLA than for an LLS, owing to the greater spatial diffusion.

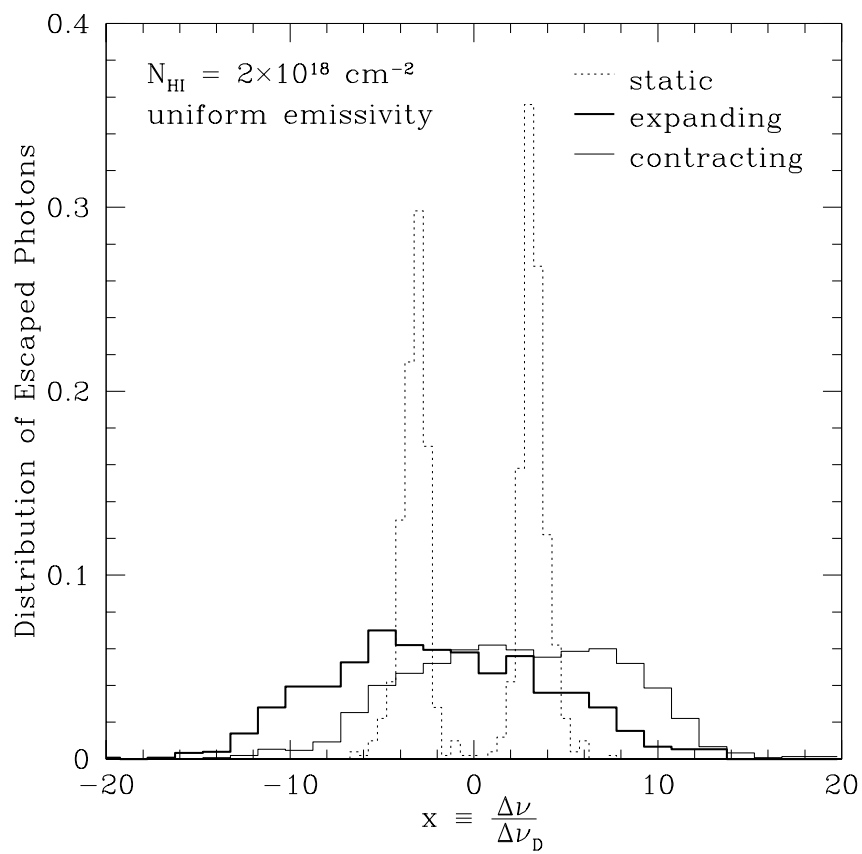

For an expanding cloud, photons should escape on average with a redshift because they are doing work on the expansion of the cloud as they are scattered. For an LLS with a central source, a photon undergoing a large positive frequency jump will be moved back to the line center relative to the fluid as it travels through the cloud, owing to the Hubble-like expansion of the cloud. On the other hand, a negative frequency jump will allow the photon to escape directly. In our case, the velocity at the cloud edge, $200 \mathrm{~km}$ $\mathrm{s}^{-1}$, is much larger than the atomic velocity dispersion, so a

FIG. 2.-Frequency distribution of Ly $\alpha$ photons that escaped from a uniform hydrogen cloud with neutral hydrogen column density $2 \times 10^{18} \mathrm{~cm}^{-2}$ for the cases of uniform emissivity and a central point source. Different line types are for a static, expanding, or contracting cloud. 

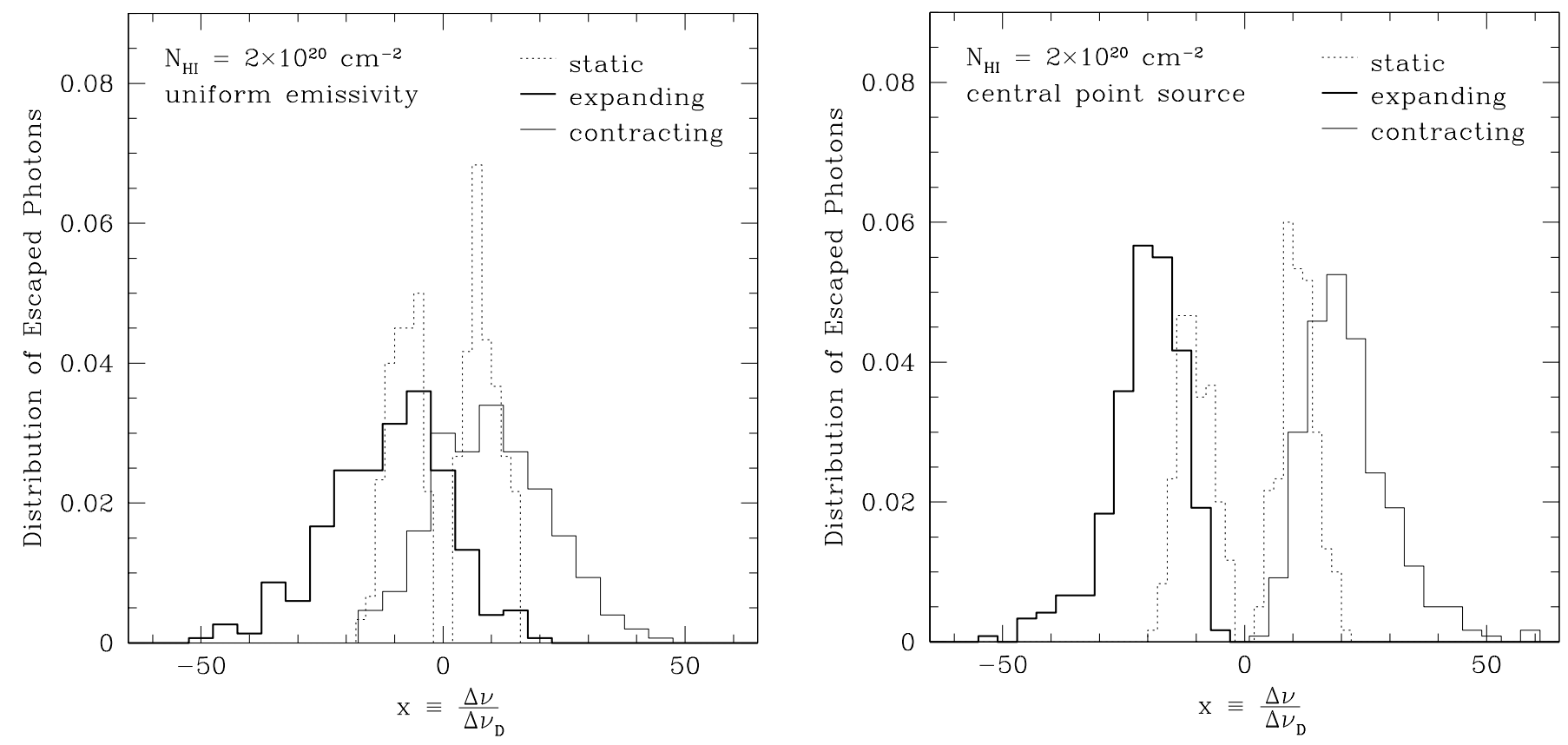

FIG. 3.-Same as Fig. 2, but for a neutral hydrogen column density of $2 \times 10^{20} \mathrm{~cm}^{-2}$

photon needs to undergo many positive frequency jumps in order to diffuse spatially through the cloud and be able to escape on the blue side of the line, which explains why the blue peak is highly suppressed (see Fig. 3 of Ahn \& Lee 1998 for a similar explanation). The situation is exactly reversed for a contracting cloud. The case of uniform emissivity broadens the line, essentially because of the different velocities of the emission sites of the photons; there is also the additional effect that photons emitted near the edge of the cloud are more likely to escape on the blue (red) peak for an expanding (contracting) cloud. In DLAs, the line is also broader for a point source compared to an LLS because of

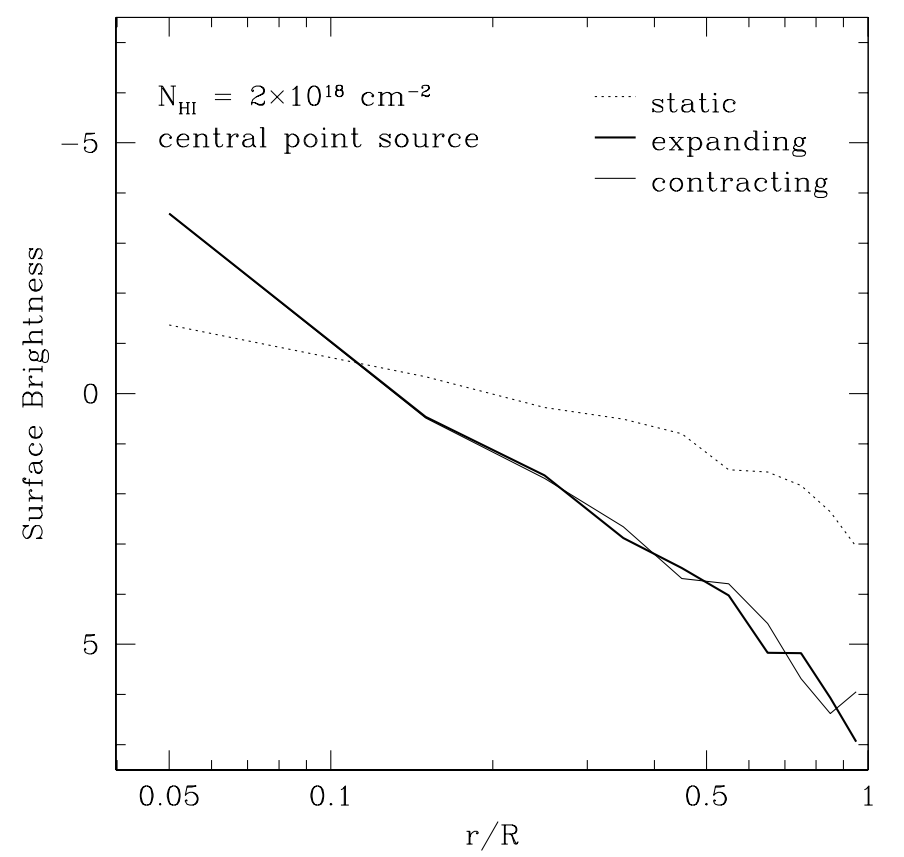

the power-law dependence of the cross section on frequency and the greater degree of spatial diffusion.

To summarize, these simple models show how the frequency and spatial distributions of Ly $\alpha$ photons escaped from a cloud are related to the $\operatorname{Ly} \alpha$ emissivity distribution, the bulk velocity field, and the column density of the cloud.

\section{Ly $\alpha$ EMISSION FROM MODEL DAMPED Ly $\alpha$ SYSTEMS}

We now apply our code to a gas cloud with an isothermal density profile as a model for DLAs. The nature of DLAs is still a subject of debate. They could be protogalaxies with a

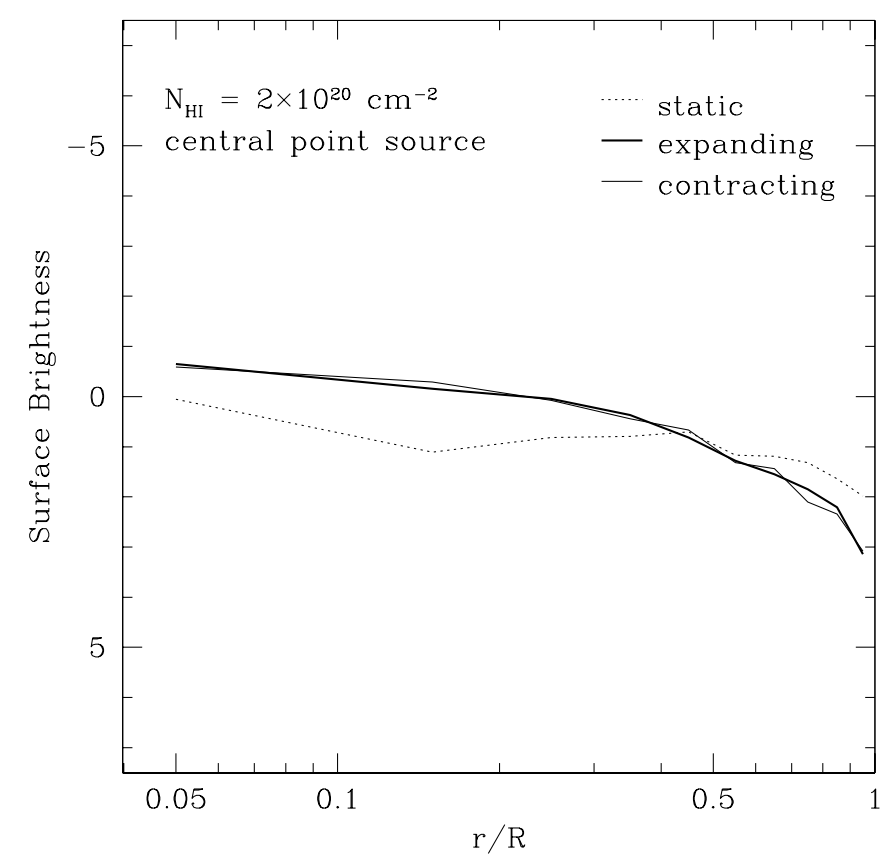

FIG. 4.- Surface brightness profiles, in magnitudes per solid angle (with an arbitrary zero point). Curves are normalized in such a way that the total energy of the photons remains the same. In the right-hand panel, the drop at $r / R \sim 0.15$ for the static case is due to simulation noise. 
rotating disk component (see, e.g., Prochaska \& Wolfe 1997, 1998) or spherically distributed clouds of gas moving randomly in halos (see, e.g., Mo 1994; Haehnelt, Steinmetz, \& Rauch 1998; McDonald \& Miralda-Escudé 1999). The other mechanism that may give rise to DLAs is large-scale outflows due to galactic winds in dwarf galaxies (see, e.g., Nulsen, Barcons, \& Fabian 1998; Tenorio-Tagle et al. 1999; Schaye 2001). In this paper, we focus on the first two pictures. We investigate the emergent spectra and spatial distribution of the Ly $\alpha$ emission to show what it can tell us about the internal structure of the system.

\subsection{Model Description}

We model the gas cloud producing a DLA assuming that it forms in a dark matter halo of mass $10^{11} M_{\odot}$, virialized at redshift $z=3$. In an $\Omega_{M}=1$ universe with $H_{0}=70 \mathrm{~km} \mathrm{~s}^{-1}$ $\mathrm{Mpc}^{-1}$, the corresponding virial radius is $r_{\mathrm{vir}}=24 \mathrm{kpc}$ and the virial velocity $V_{\text {vir }}=104 \mathrm{~km} \mathrm{~s}^{-1}$ (see, e.g., Padmanabhan 1993). We assume that the gas density profile is a singular isothermal sphere with a cutoff at the virial radius and a fraction of the halo mass in gas of 0.05 .

We consider two different cases as an illustration to show how the rotation of a system could be probed by observations of $\operatorname{Ly} \alpha$ emission. The first case is a spherically symmetric cloud and the second a rotating oblate ellipsoid with an axis ratio of 0.5 for the gas distribution. The rotating velocity is set to be $V_{\text {rot }}=(2 / 3)^{1 / 2} V_{\text {vir. In one case, we assume }}$ the gas to be static and at a temperature $2 \times 10^{4} \mathrm{~K}$ (a typical temperature of gas that cools after shock heating but stays photoionized). We also consider another case in which the gas is given an additional velocity dispersion of $V_{\text {vir }} / \sqrt{3}$ in the spherical model and $V_{\text {vir }} / 3$ in the flattened, rotating model. This additional velocity dispersion is simply added quadratically to the thermal one, which would be valid if the gas were in optically thin clumps moving at this velocity dispersion. In practice, any relevant gas clumps in DLAs will be optically thick to Ly $\alpha$ photons, but their inclusion would make our model much more complex. We will discuss the effect we would expect from optically thick clumps in $\S 5$.

There are various sources to produce Ly $\alpha$ photons: internal dissipation, star formation, and fluorescence caused by the intergalactic UV background. The external UV background will give rise to Ly $\alpha$ fluorescence; at $\sim 10^{4} \mathrm{~K}$, in an optically thick medium, recombination of the photoionized hydrogen has a $68 \%$ probability of producing a Ly $\alpha$ photon (Spitzer 1978). Shock heating of the gas will dissipate kinetic energy into heat, which will result in Ly $\alpha$ emission after line excitation and collisional ionization followed by recombination. In addition, internal star formation can of course also produce Ly $\alpha$ emission. We consider only fluorescent emission from the external background in this paper. In this case, the emissivity is proportional to the square of the ionized gas density. The other two sources of emission are more centrally concentrated, and therefore similar differences as the ones between uniform emissivity and the central source in the previous section can be expected.

We take self-shielding into account in the spherical case, calculating the neutral hydrogen density of the cloud at every radius, where the total gas density follows a singular isothermal profile. We use an iterative algorithm similar to the one presented by Tajiri \& Umemura (1998), as described in Zheng \& Miralda-Escudé (2002). Here we assume a UV background intensity of $10^{-22}$ ergs s${ }^{-1} \mathrm{~cm}^{-2} \mathrm{~s}^{-1} \mathrm{~Hz}^{-1} \mathrm{sr}^{-1}$, constant at all frequencies between the $\mathrm{H}_{\mathrm{I}}$ Lyman limit, $\nu_{\mathrm{L}}$, and the He II Lyman limit, $4 \nu_{\mathrm{L}}$. The intensity is set to zero at frequencies above $4 \nu_{\mathrm{L}}$. This is a good approximation since most higher frequency photons are likely to be absorbed by He II before reaching the hydrogen self-shielded zone. Even though in reality some very energetic photons exist, which can penetrate into the self-shielded region, their effect on the profile of neutral hydrogen is very small because of their low intensity. The profile of neutral hydrogen that is obtained is similar to that shown in Zheng \& Miralda-Escudé (2002). In the case of the flattened, rotating gas distribution, we simply take the same neutral hydrogen density profile as for the spherical case and flatten it with an axis ratio of 0.5 (the numerical code we have developed for computing the selfshielding correction applies only to spherically symmetric systems). This is of course not exact, although a much better approximation than neglecting self-shielding altogether.

\subsection{Results of Simulations}

The surface brightness profile for the spherically symmetric, nonrotating cloud is plotted in Figure 5. The thick solid line shows the case of including the fluid velocity dispersion, $\sigma=V_{\text {vir }} / \sqrt{3}=60 \mathrm{~km} \mathrm{~s}^{-1}$, while the thin solid line is the result with only the thermal velocity dispersion, $\left(k T / m_{\mathrm{H}}\right)^{1 / 2}=12.8 \mathrm{~km} \mathrm{~s}^{-1}$. The dashed line is what is obtained when self-shielding is not included (it has been shifted vertically). The velocity dispersion has practically no effect on the surface brightness profile because the change in spatial diffusion of the photons is very small. The emissivity due to the recombination has a "hole" in the inner part of the cloud because of self-shielding. Therefore, we see a "core" in the surface brightness profile (with a slight decline of surface brightness toward the center in the inner

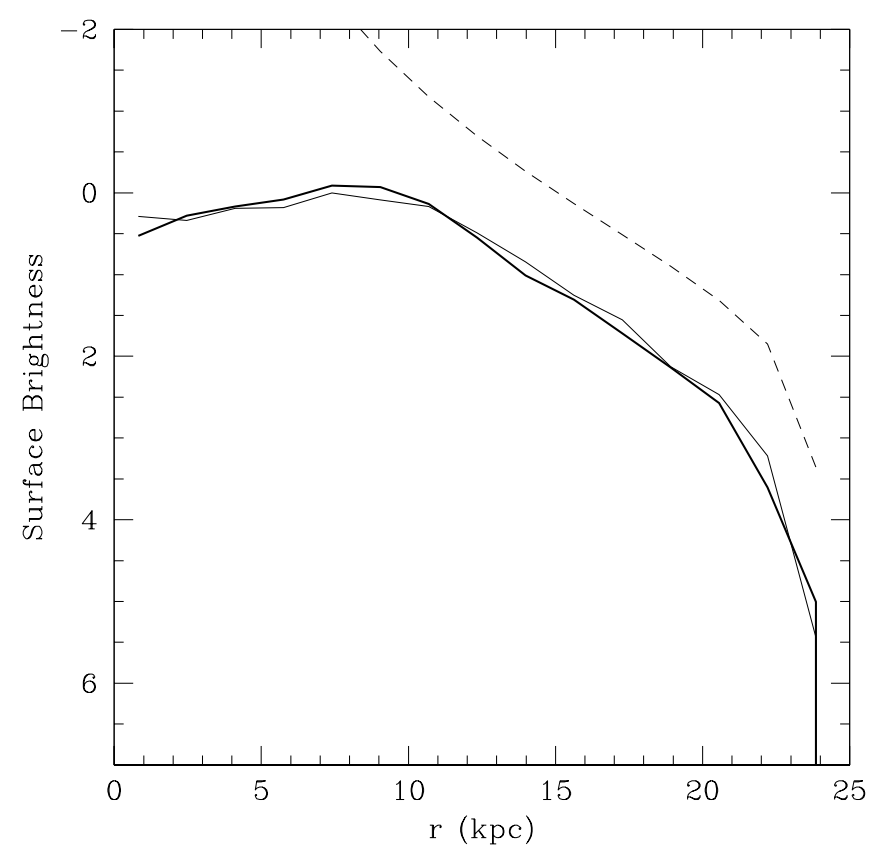

FIG. 5.-Surface brightness profiles of Ly $\alpha$ emission for the spherically symmetric cloud (in magnitudes per unit solid angle, with an arbitrary normalization). The thick and thin lines are with and without velocity dispersion, respectively (see text). The dashed line is for optically thin cloud (with an arbitrary vertical shift). 
part due to geometric effects). The outer part of the profile follows the optically thin case. The core of the surface brightness profile is a signature that the emission is due to fluorescence of the external background since other sources of emission are more centrally concentrated.

Because the ionizing photons of the external background extend only up to $4 \nu_{\mathrm{L}}$ in frequency, essentially all the photoionizations and subsequent recombinations occur in the outer region of the gas cloud with a hydrogen column density of $N_{\mathrm{HI}} \lesssim 10^{19} \mathrm{~cm}^{-2}$, the inverse of the photoionization cross section at frequency $4 \nu_{\mathrm{L}}$. The damped absorption wings are not yet important at this column density; therefore, the photons escape the cloud after a single large jump in frequency when scattered by an atom in the high-velocity tail of the Maxwellian distribution, as discussed in $\S 3$ for the case of LLSs.

Figure 6 shows that the two-dimensional spectra of the Ly $\alpha$ photons escaped from the nonrotating cloud. We assume that the slit is large enough to include the entire cloud. The double-peaked distribution of escaped Ly $\alpha$ photons, with the intermediate frequency range of essentially zero flux, appears at every spatial position.

Including an additional velocity dispersion has the effect of separating the two peaks. This is true in our model because we consider the case in which there is no correlation between the velocity of two atoms that are spatially close, valid only when any gas clumps moving coherently are so small that they are optically thin to Ly $\alpha$ photons. In other words, the additional velocity dispersion is effectively thermal. In the presence of coherent motions of optically thick regions, the intermediate frequency range with a highly reduced flux disappears, as discussed in $\S 3$ in the examples of an expanding or contracting cloud.

Spectra of the flattened rotating cloud depend on the viewing angle and slit orientation. The face-on spectrum, plotted in Figure 7, is very similar to that of the spherical static case. Figure 8 shows the edge-on spectra, with the slit perpendicular (top panels) and parallel (bottom panels) to the rotational axis. The spectrum is averaged along the spatial coordinate perpendicular to the slit; in other words, we are assuming that the entire cloud is included in the slit but that the wavelength dispersion is wide enough that the size of the cloud does not introduce any smoothing of the spectrum. When the slit is perpendicular to the rotation axis, the rotation curve pattern is clearly seen. The velocity shift of the spectrum is about the same as the rotational velocity, $V_{\text {rot }}=$ $V_{\mathrm{vir}}(2 / 3)^{1 / 2}=85 \mathrm{~km} \mathrm{~s}^{-1}$, at the edge of the system [note that the unit used in the horizontal axis is $\left.\Delta \nu_{\mathrm{D}}=\left(2 \mathrm{kT} / \mathrm{m}_{\mathrm{H}}\right)^{1 / 2} \nu_{0} / c=18.2 \mathrm{~km} \mathrm{~s}^{-1}\left(\nu_{0} / c\right)\right]$. The variation of the shape of the spectrum along the slit is easily understood by thinking of an analogy to the expanding and contracting cloud in $\S 3$. The Ly $\alpha$ photons are produced on the outer ionized layer of the system, near the radius $r_{\mathrm{ss}}$ where the hydrogen becomes self-shielding. The line-of-sight velocity at this radius is proportional to $V_{\mathrm{rot}} r_{p} / r_{\mathrm{ss}}$, where $r_{p}$ is the projected radius on the slit position. Therefore, the central trough of the spectrum shifts linearly with the projected radius. Moreover, the variation of the velocity along the line of sight causes the peak of emission that is further from the mean velocity of the system to be enhanced, for similar reasons as in the case of the expanding and contracting clouds discussed in $\S 3$.

For the case of the slit parallel to the rotational axis, the two-dimensional spectrum displays a symmetric doublepeaked pattern, which essentially results from averaging along the equator the spectrum with different projected velocity. This averaging results in a less sharp central trough and smoothing of the two peaks. In all cases, the velocity dispersion increases the distance between the two peaks in the photon distribution and broadens the width of each peak.
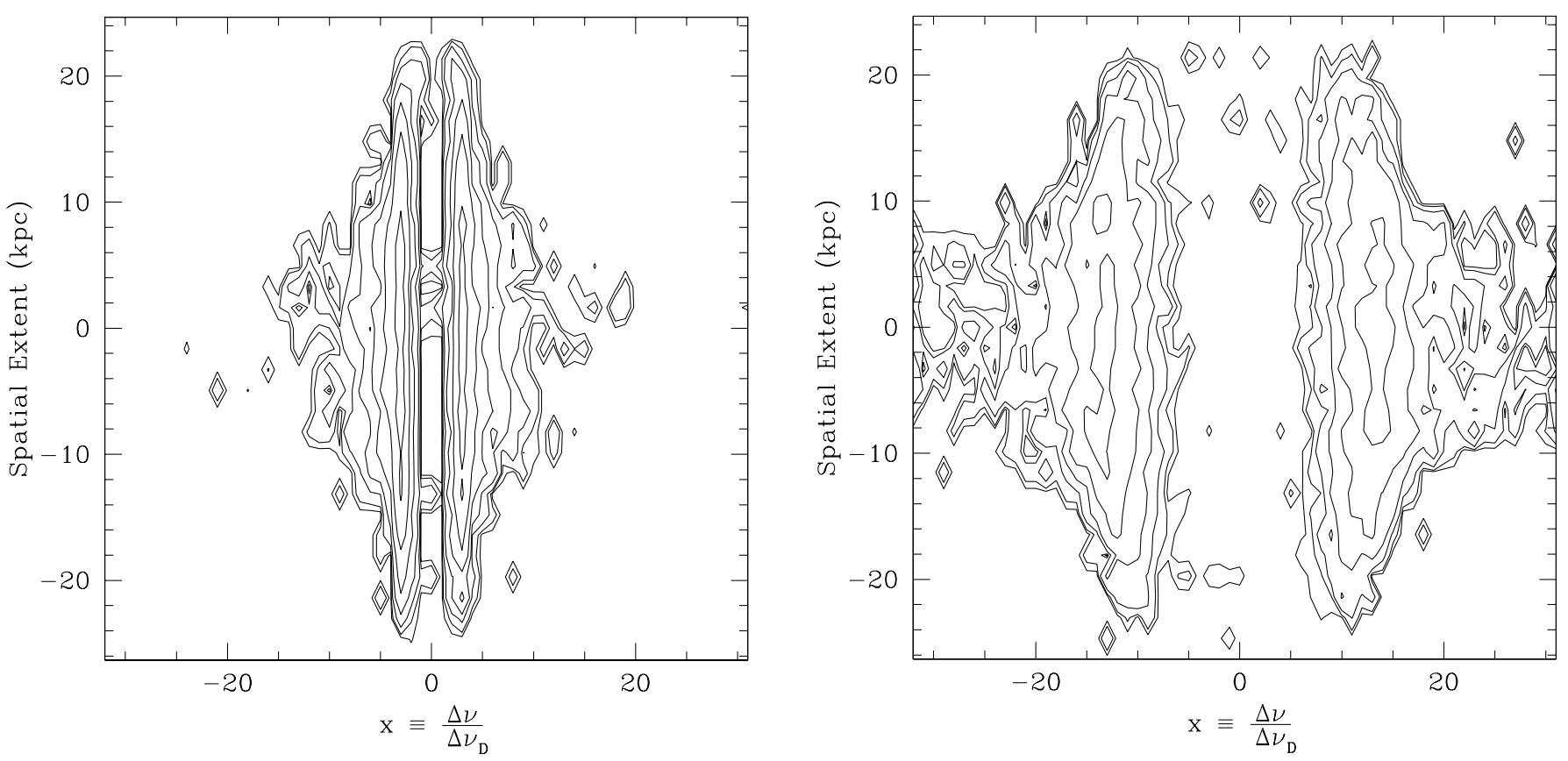

FIG. 6.-Two-dimensional spectra of the spherically symmetric, nonrotating cloud. The left-hand panel is for a thermal velocity dispersion of $\left(k T / m_{\mathrm{H}}\right)^{1 / 2}=12.8 \mathrm{~km} \mathrm{~s}^{-1}$ only; the right-hand panel includes a fluid velocity dispersion of $60 \mathrm{~km} \mathrm{~s}^{-1}$ (see text). The contours next to each other are separated by 1 mag. 

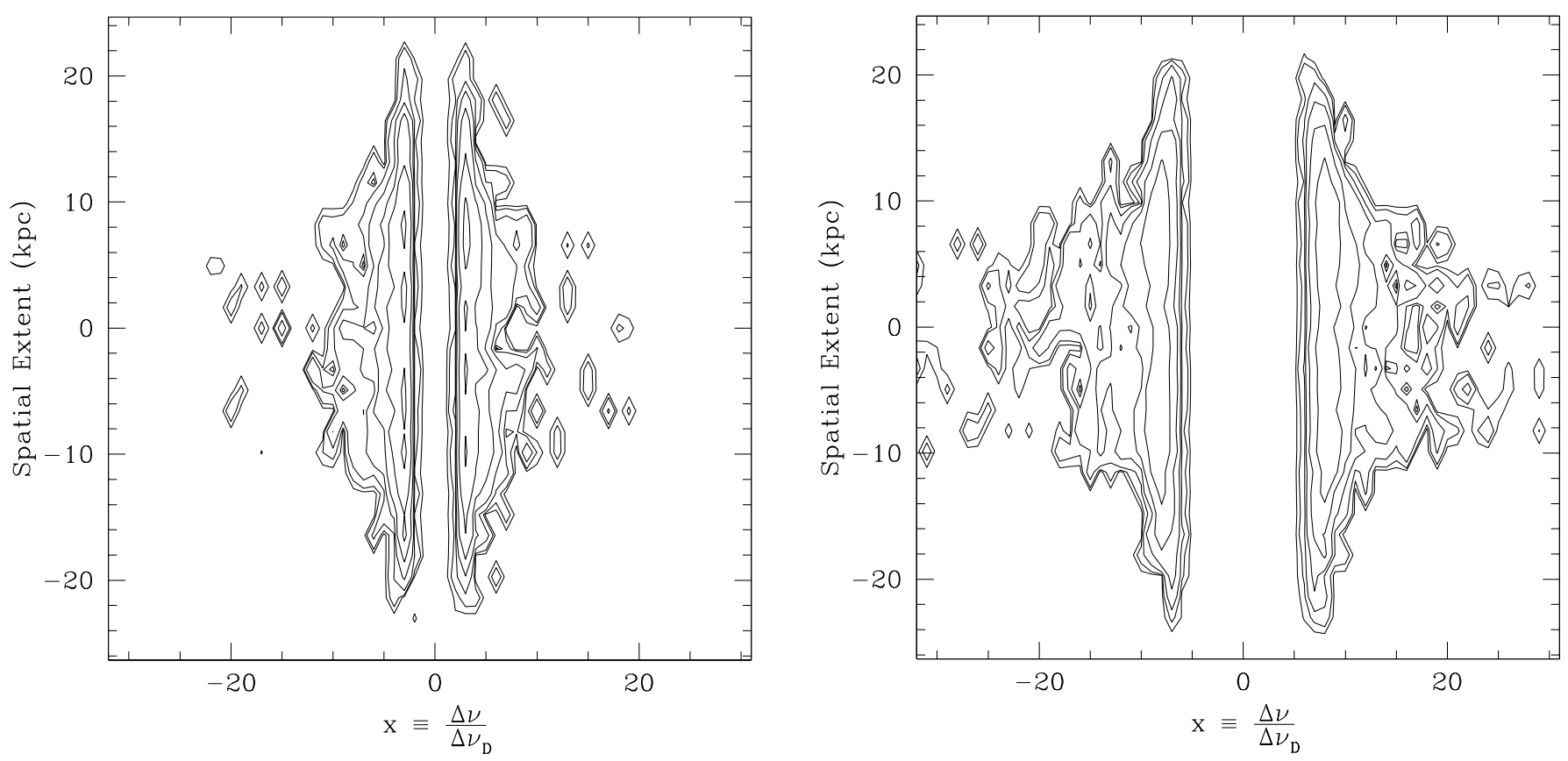

Fig. 7.-Two-dimensional spectra of the oblate rotating cloud viewed face-on. The left-hand panel is for a thermal velocity dispersion of $12.8 \mathrm{~km} \mathrm{~s} \mathrm{~s}^{-1}$ only; the right-hand panel includes an additional velocity dispersion of $35 \mathrm{~km} \mathrm{~s}^{-1}$ (see text). The contours next to each other are separated by $1 \mathrm{mag}$.

\section{SUMMARY AND DISCUSSION}

We have developed a Monte Carlo code of Ly $\alpha$ photon scattering to construct simulated images and spectra. The code is fully three-dimensional and adaptable to a cloud with any given Ly $\alpha$ emissivity, neutral hydrogen density distribution, and bulk velocity field. Several simple cases have been presented to show the images and spectra expected from a uniform gas distribution. We have applied the code to model DLAs illuminated by the intergalactic UV background to study the spatial and frequency distribution of emitted Ly $\alpha$ photons. Self-shielding produces a core in the Ly $\alpha$ surface brightness profile. For a spherical, static cloud, Ly $\alpha$ photons have a double-peaked distribution that is symmetric around the line-center frequency. An oblate, rotating cloud shows the same double-peaked distribution with the rotational pattern of increasing mean wavelength with the position on the slit. Observations of the fluorescent emission from DLAs with large telescopes (Hogan \& Weymann 1987; Gould \& Weinberg 1996) could reveal the presence of such systems and measure a velocity gradient, providing a direct measurement of the rotation rate. The Ly $\alpha$ image would also reveal if the emission is due to fluorescence from the external radiation (in which case we expect a large core of the surface brightness at the radius where the hydrogen becomes self-shielded) or to internal gas dissipation or star formation.

The observations of metal lines in DLAs show multiple absorption lines (see, e.g., Prochaska \& Wolfe 1997, 1998), suggesting that the gas is clumpy. In this paper, we have assumed instead that the gas has a smooth distribution. The case of enhanced velocity dispersion we have considered is valid only if the gas is in very small clumps that are optically thin to Ly $\alpha$ photons. Clumps that can give rise to the observed metal lines are highly optically thick. The effect of a clumpy gas distribution is not difficult to imagine. If there is only one clump along a line of sight, then this clump will essentially produce the same spectral shape in its emission line, shifted to its velocity. Thus, if the emission line could be observed with sufficient angular resolution to resolve the individual clumps, the characteristic spectral shape of Figure 6 would appear at every position, although shifted to the clump velocity. If the clumps are not resolved, the emission-line shape is obviously smoothed, just like in the case of an expanding or contracting cloud shown in Figure 2. Our numerical code can be applied in the future to the results of detailed hydrodynamic simulations of gaseous halos in the process of forming galaxies in cosmological realizations to predict other observable signatures that can test the mechanisms by which energy dissipation of gas leads to galaxy formation.

A possible effect that we have not considered in this paper is the absorption of Ly $\alpha$ photons by dust. Dust seems to be present in at least some DLAs as indicated by the reddening of the background quasars that have DLAs along the line of sight (Fall, Pei, \& McMahon 1989; Pei, Fall, \& Bechtold 1991) and the abundance ratios of iron-peak elements that follow a dust depletion pattern (Savage \& Sembach 1996; Vladilo 1998). The resonant nature of the scattering of Ly $\alpha$ photons by neutral hydrogen atoms increases the probability for them to be absorbed by dust grains. This is probably relatively more important when the Ly $\alpha$ emission originates from a star-forming region (see, e.g., Meier \& Terlevich 1981; Charlot \& Fall 1993), where dust is likely to be abundant. If Ly $\alpha$ photons of DLAs are caused by fluorescent emission, like cases we consider in this paper, the absorption by dust would not be very severe because of the low column densities of the ionized outer layer (Charlot \& Fall 1991). Since in the presence of dust the escape of $\operatorname{Ly} \alpha$ photons depends on many factors, such as the $\mathrm{H}$ I column density, the distribution of $\operatorname{Ly} \alpha$ source, the property of dust, and the 

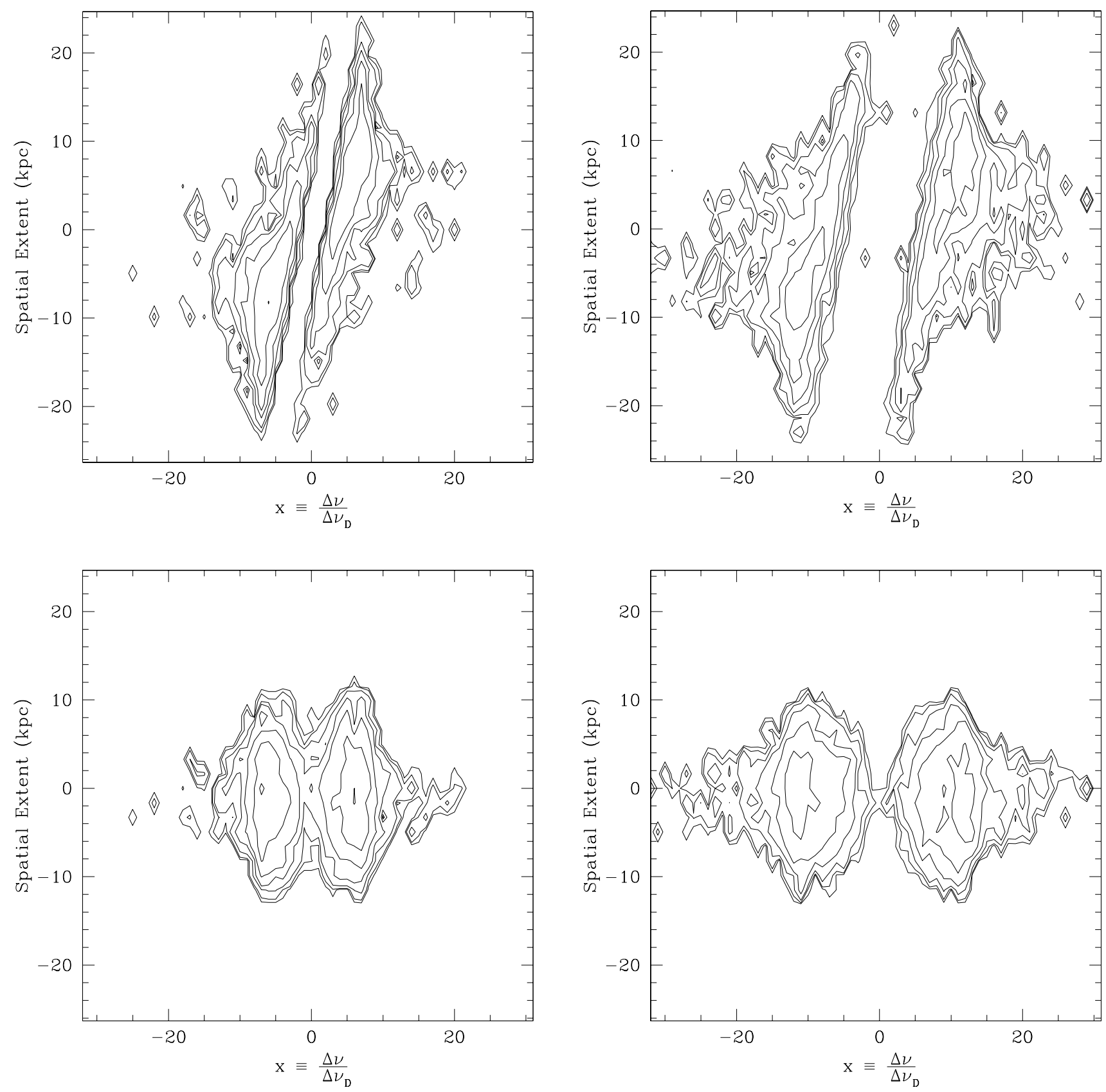

FIG. 8. - Two-dimensional spectra of the oblate rotating cloud viewed edge-on, with the slit perpendicular (top) and parallel (bottom) to the rotation axis. The left- and right-hand panels are for cases of different velocity dispersions as explained in Fig. 7 . The contours next to each other are separated by 1 mag.

topology of the medium (see, e.g., Ferland \& Netzer 1979; Hummer \& Kunasz 1980; Neufeld 1990, 1991; Charlot \& Fall 1991), detailed modeling of DLAs is necessary for dust absorption to be taken into consideration.
The authors thank the referee Joop Schaye for helpful comments. We also thank Xuelei Chen and David Weinberg for useful discussions. This work was supported in part by NSF grant NSF 0098515.

\section{APPENDIX}

To choose the velocity along the direction of the incident photon of the atom responsible for the scattering, we need to generate a random number $u$ that has the following distribution (see eq. [4]):

$$
f(u) \propto \frac{e^{-u^{2}}}{(x-u)^{2}+a^{2}},
$$


where $x$ and $a$ are given. We give a brief description on how we generate random numbers with this distribution. Since the distribution has the symmetry under the transformation of $(x, u) \rightarrow(-x,-u)$, we limit the following discussion to $x>0$.

We make use of the rejection method (Press et al. 1992) to generate $u$. The comparison distribution can be chosen to be $g(u) \propto\left[(x-u)^{2}+a^{2}\right]^{-1}$, which can be integrated and inverted analytically. A value of $u$ with the distribution $g(u)$ is first generated. We keep this value only if a second random number uniformly distributed between 0 and 1 is smaller than $e^{-u^{2}}$. In this way, we obtain values of $u$ with the distribution $f(u)$. In practice, when $x \gg 1$, the above method is inefficient because a very small fraction of values of $u$ are not discarded. To increase the fraction of acceptance, we modify the comparison distribution to be

$$
g(u) \propto \begin{cases}{\left[(x-u)^{2}+a^{2}\right]^{-1},} & u \leq u_{0}, \\ e^{-u_{0}^{2}}\left[(x-u)^{2}+a^{2}\right]^{-1}, & u>u_{0} .\end{cases}
$$

The value of $u_{0}$ can be chosen to minimize the fraction of generated values that will be discarded. The acceptance fractions are then required to be $e^{-u^{2}}$ and $e^{-u^{2}} / e^{-u_{0}^{2}}$ in the regions $u \leq u_{0}$ and $u>u_{0}$, respectively.

A first random number $R$ uniformly distributed between 0 and 1 determines which region we use by comparing it with $p$, where

$$
p=\frac{\int_{-\infty}^{u_{0}} g(u) d u}{\int_{-\infty}^{+\infty} g(u) d u}=\left(\theta_{0}+\frac{\pi}{2}\right)\left[\left(1-e^{-u_{0}^{2}}\right) \theta_{0}+\left(1+e^{-u_{0}^{2}}\right) \frac{\pi}{2}\right]^{-1}, \quad \theta_{0}=\tan ^{-1} \frac{u_{0}-x}{a} .
$$

We generate $u$ through $u=a \tan \theta+x$, where $\theta$ is a random number uniformly distributed in $\left[-\pi / 2, \theta_{0}\right]$ and $\left[\theta_{0}, \pi / 2\right]$ for $R \leq p$ and $R>p$, respectively. Then another random number uniformly distributed between 0 and 1 determines whether the generated value of $u$ is accepted by comparing it with the corresponding fraction of acceptance.

\section{REFERENCES}

Adams, T. F. 1972, ApJ, 174, 439

Ahn, S.-H., \& Lee, H.-W. 1998, preprint (astro-ph/9801031)

Ahn, S.-H., Lee, H.-W., \& Lee, H.-M. 2000, J. Korean Astron. Soc, 33, 29 2001, ApJ, 554, 604 2002, ApJ, 567, 922

Auer, L. H. 1968, ApJ, 153, 783

Avery, L. W., \& House, L. L. 1968, ApJ, 152, 493

Bonilha, J. R. M., Ferch, R., Salpeter, E. E., Slater, G., \& Noerdlinger, P. D. 1979, ApJ, 233, 649

Briggs, F. H., Wolfe, A. M., Liszt, H. S., Davis, M. M., \& Turner, K. C. 1989, ApJ, 341, 650

Caroff, L. J., Noerdlinger, P. K., \& Scargle, J. D. 1972, ApJ, 176, 439

Charlot, S., \& Fall, S. M. 1991, ApJ, 378, 471

. 1993, ApJ, 415, 580

Djorgovski, S. G. 1998, in Structure and Evolution of the IGM from QSO

Absorption Lines, ed. P. Petitjean \& S. Charlot (Paris: Editions Frontières), 303

Djorgovski, S. G., Pahre, M. A., Bechtold, J., \& Elston, R. 1996, Nature, 382,234

Fall, S. M., Pei, Y. C., \& McMahon, R. G. 1989, ApJ, 341, L5

Ferland, G., \& Netzer, H. 1979, ApJ, 229, 274

Field, G. 1959, ApJ, 129, 551

Fynbo, J. U., Møller, P., \& Warren, S. J. 1999, MNRAS, 305, 849

Fynbo, J. U., Thomsen, B., \& Møller, P. 2000, A\&A, 353, 457

Gould, A., \& Weinberg, D. H. 1996, ApJ, 468, 462

Haehnelt, M. G., Steinmetz, M., \& Rauch, M. 1998, ApJ, 495, 647

Harrington, J. P. 1973, MNRAS, 162, 43 . 1974, MNRAS, 166, 373

Hogan, C. J., \& Weymann, R. J. 1987, MNRAS, 225, P1

Hummer, D. G., \& Kunasz, P. B. 1980, ApJ, 236, 609

Hunstead, R. W., Pettini, M., \& Fletcher, A. B. 1990, ApJ, 356, 23

Leibundgut, B., \& Robertson, G. 1999, MNRAS, 303, 711

Loeb, A., \& Rybicki, G. B. 1999, ApJ, 524, 527
Lu, L., Sargent, W. L. W., Barlow, T. A., Churchill, C. W., \& Vogt, S. S. 1996, ApJS, 107, 475

McDonald, P., \& Miralda-Escudé, J. 1999, ApJ, 519, 486

Meier, D. L., \& Terlevich, R. 1981, ApJ, 246, L109

Mo, H. J. 1994, MNRAS, 269, L49

Natta, A., \& Beckwith, S. 1986, A\&A, 158, 310

Neufeld, D. A. 1990, ApJ, 350, 216

. 1991, ApJ, 370, L85

Nulsen, P. E. J., Barcons, X., \& Fabian, A. C. 1998, MNRAS, 301, 168

Osterbrock, D. E. 1962, ApJ, 135, 195

Padmanabhan, T. 1993, Structure Formation in the Universe (Cambridge: Cambridge Univ. Press)

Panagia, N., \& Ranieri, M. 1973, A\&A, 24, 219

Pei, Y. C., Fall, S. M., \& Bechtold, J. 1991, ApJ, 378, 6

Pettini, M., Hunstead, R. W., King, D. L., \& Smith, L. J. 1995, in QSO Absorption Lines, ed. G. Meylan (Berlin: Springer), 55

Press, W. H., Teukolsky, S. A., Vetterling, W. T., \& Flannery, B. P. 1992, Numerical Recipes (Cambridge: Cambridge Univ. Press)

Prochaska, J. X., Gawiser, E., \& Wolfe, A. M. 2001, ApJ, 552, 99

Prochaska, J. X., \& Wolfe, A. M. 1997, ApJ, 487, 73

. 1998, ApJ, 507, 113

Savage, B. D., \& Sembach, K. R. 1996, ARA\&A, 34, 279

Schaye, J. 2001, ApJ, 559, L1

Spitzer, L. 1978, Physical Processes in the Interstellar Medium (New York: Wiley)

Tajiri, Y., \& Umemura, M. 1998, ApJ, 502, 59

Tenorio-Tagle, G., Silich, S. A., Kunth, D., Terlevich, E., \& Terlevich, R. 1999, MNRAS, 309, 332

Urbaniak, J. J., \& Wolfe, A. M. 1981, ApJ, 244, 406

Vladilo, G. 1998, ApJ, 493, 583

Warren, S., \& Møller, P. 1996, A\&A, 311, 25

Wouthuysen, S. A. 1952, AJ, 57, 31

Zheng, Z., \& Miralda-Escudé, J. 2002, ApJ, 568, L71 